\title{
Inhibitory effect of tanshinone IIA on inflammatory response in rheumatoid arthritis through regulating $\beta$-arrestin 2
}

\author{
JING TANG $^{1 *}$, SIWEI ZHOU ${ }^{1 *}$, FANGHUA ZHOU ${ }^{1}$ and XIUMEI WEN ${ }^{2}$ \\ ${ }^{1}$ Orthopaedics Rehabilitation Center, Zhejiang Rehabilitation Medical Center, Hangzhou, \\ Zhejiang 310051; ${ }^{2}$ Department of Emergency, The Third Affiliated Hospital of Zhejiang \\ Chinese Medical University, Hangzhou, Zhejiang 310005, P.R. China
}

Received November 2, 2017; Accepted August 31, 2018

DOI: $10.3892 /$ etm.2019.7371

\begin{abstract}
The current study aimed to investigate the inhibitory effect of tanshinone IIA (Tan IIA) on the inflammatory response in patients with rheumatoid arthritis (RA) and explore its mechanism. A total of 50 patients with RA were randomly separated into the control group (15 cases) and the research group (35 cases). The tumor necrosis factor (TNF)- $\alpha$ and interleukin (IL)-6 levels in serum were determined, and peripheral blood mononuclear cells (PBMCs) were separated from patients with RA and cultured in vitro. The effects of the $\beta$-arrestin 2 small interfering (si)RNA incubation, lipopolysaccharide (LPS) stimulation or Tan IIA incubation on TNF- $\alpha$ and IL- 6 levels, and the expression levels of $\beta$-arrestin 2 , NAD-dependent protein deacetylase sirtuin-1 (SIRT1) and transcription factor p65 (p65) proteins were investigated. Prior to treatment, no significant differences in TNF- $\alpha$ and IL-6 levels in serum of patients with RA were identified between the research and control groups. Following treatment, the TNF- $\alpha$ and IL- 6 levels in the serum of patients with RA in the research group were significantly lower compared with those in the research group prior to treatment and those in the control group following treatment $(\mathrm{P}<0.05)$. Tan IIA inhibited the LPS-induced secretion of TNF- $\alpha$ and IL-6, upregulated the LPS-inhibited expression of the $\beta$-arrestin 2 and SIRT1 proteins, and downregulated the LPS-induced expression of the p65 protein in the PBMCs of patients with RA. The $\beta$-arrestin 2 small interfering (si)RNA significantly upregulated the secretion of TNF- $\alpha$ and IL-6, inhibited the expression of the SIRT1 protein and upregulated the expression of the
\end{abstract}

Correspondence to: Dr Xiumei Wen, Department of Emergency, The Third Affiliated Hospital of Zhejiang Chinese Medical University, 219 Moganshan Road, Hangzhou, Zhejiang 310005, P.R. China

E-mail: wenxmtahzjcmu@163.com

${ }^{*}$ Contributed equally

Key words: tanshinone IIA, rheumatoid arthritis, inflammatory response, $\beta$-arrestin 2 p65 protein in PBMCs of patients with RA. Tan II A effectively increased the weight of rats with rheumatoid arthritis, and reduced the circumference of the left posterior ankle, the posterior plantar metatarsal thickness, and the content of serum TNF- $\alpha$ and IL-6. Tan IIA did not significantly reverse these $\beta$-arrestin 2 siRNA-induced changes. Tan IIA inhibited the inflammatory response in PBMCs of patients with RA by upregulating $\beta$-arrestin 2 expression.

\section{Introduction}

The key clinical features of rheumatoid arthritis (RA), an inflammatory autoimmune disease, are synovial inflammation and chronic corrosive damage to joints; however, its pathogenesis is unknown at present (1-3). In patients with RA, synovial hyperplasia and tissue inflammation may cause joint and surrounding tissue damage, resulting in joint deformity and dysfunction (4,5). Furthermore, lesions may also affect all joints with synovial membranes, with those of the hands and feet being the most common $(4,5)$. Several studies have indicated that inflammatory cytokines and chemokines, including tumor necrosis factor (TNF)- $\alpha$, interleukin (IL)- 6 and IL-1 $\beta$ serve key roles in the development and progression of autoimmune diseases, including systemic lupus erythematosus, systemic vasculitis, scleroderma and RA (1-3). Humanized anti-TNF- $\alpha$ (4) and anti-IL-6 receptor (5) monoclonal antibodies, and inflammatory cytokines or chemokines antagonist drugs are widely used to treat RA in clinic; these treatments demonstrate good clinical therapeutic effects on RA (5). In addition, certain therapeutic drugs that target immune cells have also been developed and applied clinically, including the CTLA4-IgG1 fusion protein, which was developed by Repligen and was approved for clinical use in the treatment of graft-versus-host responses (6). Furthermore, CD2 receptor antibodies and anti-T-cells are also at different stages of clinical research $(7,8)$.

Therefore, it is feasible to treat RA by inhibiting the inflammatory response.

Tanshinone IIA (Tan IIA), the main active ingredient in tanshinone, can effectively inhibit the lipopolysaccharide (LPS)-induced expression of pro-inflammatory molecules in human peripheral blood mononuclear cells (PBMCs) (9), downregulate serum levels of inflammatory cytokine in 
coronary syndromes (10) and induces apoptosis in fibroblast-like synoviocytes in RA (11). However, the effect of Tan IIA on the inflammatory response in patients with RA and the corresponding mechanism of action remain unknown. Therefore, the aim of the current study was to investigate the inhibitory effect of Tan IIA on the inflammatory response in patients with RA and explore the corresponding mechanism by investigating the effect of Tan IIA on the LPS-induced expression of associated proteins [sirtuin-1 (SIRT-1) p65 and $\beta$-arrestin2] in PBMCs, and TNF- $\alpha$ and IL-6 levels. The current study also aimed to assess whether Tan IIA inhibits the inflammatory response in patients with RA by inhibiting the expression of various inflammatory factors (including TNF- $\alpha$ and IL-6) in PBMCs.

\section{Materials and methods}

Clinical data. The diagnosis of RA was performed according to the ACR/EULAR 2010 classification criteria (12). A total of 50 randomly selected patients with RA were recruited from the Department of Rheumatology, Third Affiliated Hospital of Zhejiang Chinese Medical University (Hangzhou, China) between January and June 2016. A total of 35 patients with RA, including 10 males and 25 females, with a mean age of $50.3 \pm 9.8$ years and a mean progression time of $6.9 \pm 2.3$ years were recruited as the research group. A total of 15 patients with RA, including 5 males and 10 females, with a mean age of $49.7 \pm 7.6$ years and a mean progression time of $6.8 \pm 1.9$ years were recruited as the control group. No significant differences in the age, gender, progression time and other general data were identified between the research and control groups. The current study was approved by the Ethics Committee of The Third Affiliated Hospital of Zhejiang Chinese Medical University. Written informed consent was obtained from each patient. The exclusion criteria were as follows: i) Patients with RA who also had other rheumatism diseases, immune system diseases, tumors and liver and kidney function deficiencies; ii) patients with RA who were pregnant, lactating or had mental diseases; and iii) patients with RA who used immunomodulatory drugs within half a year.

Clinical drugs and experimental materials. Methotrexate (product ID: H31020644) and meloxicam (product ID: H20030486) were purchased from Shanghai Sine Pharma Co., Ltd. (Shanghai, China) and Simcere Pharmaceutical Co., Ltd. (Nanjing, China), respectively. A Tan IIA sulfonate sodium injection (product ID: H31022558) and leflunomide (product ID: H20080420) were purchased from Shanghai No. 1 Biochemical \& Pharmaceutical Co., Ltd. (Shanghai, China) and Jiangsu Yabang Aipusen Pharmaceutical Co., Ltd. (Yancheng, China), respectively. Tan IIA was purchased from Sigma-Aldrich; Merck KGaA (Darmstadt, Germany). TNF- $\alpha$ (cat. no. KLC103a.96) and IL-6 (cat. no. AF-200-06) ELISA kits were purchased from Shanghai Kang Lang Biological Technology Co., Ltd. (Shanghai, China) and Shanghai Institute of Biological Products Co., Ltd. (Shanghai, China), respectively. A rat TNF- $\alpha$ ELISA kit (cat. no. K1052-100) was purchased from Amyjet Scientific Inc. (Wuhan, China). A rat IL-6 ELISA kit (cat. no. KS12244) was purchased from Shanghai Keshun Biological Technology Co., Ltd. (Shanghai,
China). An Animal tissue/cell total protein extraction kit (cat. no. SD-001/SN-002) was purchased from Amyjet Scientific, Inc.

Cell transfection. An siRNA negative control (F:5'-CCCAUU CAUUGUUGUCACUTT-3', R:5'-AGUGACAACAAUGAA UGGGTT-3'), a $\beta$-arrestin 2 siRNA oligo sequence (oligol, 5'-ACCUUUUCGUCUUUUGCUCCC-3'; oligo2, 5'-GAG CAAAAGACGAAAAGGUUG-3') were synthesized by Shanghai Gene Pharma Co., Ltd. (Shanghai, China). These were directly transferred into cells via the Lipofectamine ${ }^{\mathrm{TM}}$ 2000 transfection reagent (cat. no. 11668019; Invitrogen; Thermo Fisher Scientific, Inc.). The final concentration of siRNA used was $50 \mathrm{nM}$.

Rheumatoid rat model. A total of 60 male Sprague Dawley rats (age, 5-6 weeks; weight, 200-220 g) were purchased from Shanghai SLAC Laboratory Animal Co., Ltd. (Shanghai, China). Animals were housed at a temperature of $20-24^{\circ} \mathrm{C}$ and a humidity of $60 \%$ with a $12 \mathrm{~h}$ light/dark cycle for one week. Rats were able to drink and eat freely prior to and during the experiment. A total of 10 rats were selected as the control group. The skin of left posterior foot on each rat was sterilized with $75 \%$ alcohol and iodophor disinfectant, and $0.1 \mathrm{ml}$ saline was injected. The other 50 rats were selected as the model group. The procedure for the model group was as follows: Heat inactivated bacillus Calmette-Guérin and Freund's adjuvant (cat. no. YS-XQ0496; Shanghai Yansheng Biochemical Reagent Co., Ltd., Shanghai, China) in a $10 \mathrm{mg} / \mathrm{ml}$ oil and water emulsion was obtained from an ice bath, and then the skin of left posterior foot on each rat was sterilized with $75 \%$ alcohol and iodophor disinfectant and $0.1 \mathrm{ml}$ prepared emulsion was injected. After 14 days, the rats in model group were again injected with $0.1 \mathrm{ml}$ prepared emulsion.

Experimental animal administration and collection of specimens. Following the establishment of the RA model, the rats were fed for 1 week. Then the RA model rats were separated into the following three groups: Control model (RA), routine treatment (RT) and Tan IIA-treated (Tan IIA). The control group and RA groups were provided a normal diet. Saline $(1 \mathrm{ml} / 100 \mathrm{~g})$ was given by gavage administration once daily for 3 weeks. The RT group was fed a normal diet; the rats were also administered with methotrexate tablets dissolved in physiological saline by gavage $(0.51 \mathrm{mg} / \mathrm{kg} /$ day $)$, once every 3 days for 3 weeks. The Tan IIA group was fed a normal diet; the rats were also administered with Tan IIA dissolved in physiological saline by gavage $(0.50 \mathrm{mg} / \mathrm{kg} / \mathrm{d})$, once every 3 days for 3 weeks.

After 3 weeks, the weights of the rats was recorded prior to and following the 12 -h fasting state. Rats were anesthetized with chloral hydrate $(400 \mathrm{mg} / \mathrm{kg}$, intraperitoneal; Sinopharm Chemical Reagent Co., Ltd., Shanghai, China) and blood was extracted from the heart of each rat. Rats were subsequently sacrificed by cervical dislocation. Blood $(5 \mathrm{ml})$ was extracted using a vacuum chamber without anticoagulants for $30 \mathrm{~min}$ in a $37^{\circ} \mathrm{C}$ constant temperature water bath. The blood was then centrifuged at a speed of $500 \mathrm{x} \mathrm{g}$ for $10 \mathrm{~min}$ at room temperature to separate the serum. The serum was then divided into $100 \mu \mathrm{l}$ tubes and preserved at $4^{\circ} \mathrm{C}$. 
General examination of different groups. Three variables were measured $0,14,21,28,35$ and 42 days after the establishment of the RA model. The variables were as follows: Weight, perimeter of the left posterior ankle and posterior plantar metatarsal thickness.

Clinical treatment methods and detection indices. Patients in the research and control groups orally received conventional drug treatment, including $5 \mathrm{mg}$ methotrexate once every 2 weeks and $7.5 \mathrm{mg}$ meloxicam and $10 \mathrm{mg}$ leflunomide once daily. Patients in the research group received a Tan IIA injection of $40 \mathrm{mg}$ via an intravenous drip. Prior to treatment and following 1 week of continuous treatment, $10 \mathrm{ml}$ venous blood was obtained from all patients; the blood was centrifuged at $500 \mathrm{x} \mathrm{g}$ for $10 \mathrm{~min}$ at room temperature to obtain serum. TNF- $\alpha$ and IL- 6 serum levels were determined using ELISA kits following the manufacturer's protocol.

Separation and culture of PBMCs. Prior to treatment, venous blood was obtained from patients with RA in the control group and used to obtain PBMCs by density gradient centrifugation (1,000 x $\mathrm{g}$ for $25 \mathrm{~min}$ at room temperature). Two PBMCs samples $\left(3-5 \times 10^{6}\right.$ cells) were obtained from each patient and cultured at $37^{\circ} \mathrm{C}$ in RPMI-1640 (cat. no. 12491-15; Thermo Fisher Scientific, Inc., Waltham, MA, USA) supplemented with $10 \%$ fetal bovine serum (FBS; cat. no. 10100-147; Thermo Fisher Scientific, Inc.). Subsequently, one cultured PBMC sample was treated with $1 \mu \mathrm{g} / \mathrm{ml}$ LPS (cat. no. 00-4976-93; Thermo Fisher Scientific, Inc.) for $48 \mathrm{~h}$ at $37^{\circ} \mathrm{C}$. The other sample was treated with $1 \mu \mathrm{g} / \mathrm{ml}$ LPS for $24 \mathrm{~h}$ at $37^{\circ} \mathrm{C}$, then $0.75 \mu \mathrm{g} / \mathrm{ml}$ Tan IIA was added for a further $24 \mathrm{~h}$ of induction at $37^{\circ} \mathrm{C}$. Subsequently, both PBMCs samples were centrifuged (at $500 \mathrm{x} \mathrm{g}$ for $5 \mathrm{~min}$ at room temperature) to obtain the supernatant and PBMCs. TNF- $\alpha$ and IL- 6 serum levels were determined using ELISA kits in accordance with the manufacturer's protocol. The total proteins of PBMCs were extracted using total cell protein extraction kit and stored at $-80^{\circ} \mathrm{C}$ prior to analysis.

siRNA interference assay. A total of $5 \mathrm{ml}$ PBMCs $\left(1.0-1.5 \times 10^{6} / \mathrm{ml}\right)$ were conventionally cultured and centrifuged $(500 \mathrm{x} \mathrm{g}$ for $5 \mathrm{~min}$ at room temperature) to remove medium. Following one wash of the cells in PBS, PBMCs were treated with Lipofectamine 2000 transfection reagent kit (cat. no. 11668019; Thermo Fisher Scientific, Inc.) to transfect $\beta$-arrestin 2 siRNA (100 nM). PBMCs treated with $\beta$-arrestin 2 siRNA were regarded as the control group. After transfecting the cells for $4 \mathrm{~h}$, the medium (RPMI 1640 and 10\% FBS) was refreshed. After incubating the cells for $24 \mathrm{~h}, 1 \mu \mathrm{g} / \mathrm{ml} \mathrm{LPS}$ was added to the medium for another $24 \mathrm{~h}$. Then $0.75 \mu \mathrm{g} / \mathrm{ml}$ Tan IIA was or was not added to the PBMCs for another 24 h. Subsequently, the PBMCs samples were centrifuged (at $500 \mathrm{x}$ g for $5 \mathrm{~min}$ at room temperature) to obtain supernatant and PBMCs. TNF- $\alpha$ and IL-6 serum levels were determined using ELISA kits. The total proteins of PBMCs were extracted using total cell protein extraction kit and stored at $-80^{\circ} \mathrm{C}$ until analysis.

Western blot analysis. Protein was extracted using RIPA (cat. no. P0013C; Beyotime Institute of Biotechnology, Haimen,
China) and the concentration of total PBMC protein was determined using a BCA protein assay kit. Then total protein (70 $\mu \mathrm{g}$ /lane) was separated using SDS-PAGE (5\% gel for concentration and $12 \%$ gel for separation) and transferred to a polyvinylidene difluoride membrane (cat. no. LC2002; Thermo Fisher Scientific, Inc.). Following blocking at room temperature with $5 \%$ bovine serum albumin for $1 \mathrm{~h}$ (cat. no. 30036727; Thermo Fisher Scientific, Inc.), the membrane was incubated with primary and second antibodies. Following incubation with BeyoECL plus (cat. no. p0018M; Beyotime Institute of Biotechnology), the protein bands were analyzed by Image J software (National Institutes of Health, Bethesda, MD, USA). The primary antibody included anti- $\beta$-arrestin 2 (cat. no. ab54790), anti-NAD-dependent protein deacetylase SIRT1 (19A7AB4; cat. no. ab110304; both 1:2,000), anti-transcription factor p65 (p65; cat. no. ab16502; 1:1,000) and anti- $\beta$-actin (cat. no. ab8227; 1:2,000; Abcam, Cambridge, UK) antibodies. The secondary antibodies included horseradish-peroxidase-conjugated goat anti-mouse (cat. no. ab6789) and anti-rabbit (cat. no. ab6721; both 1:2,000; Abcam) antibodies.

Detection of $m R N A$ expression by semi-quantitative reverse transcription polymerase chain reaction $(R T-P C R)$ analysis. Total RNA was extracted from PBMCs using cell RNA kit (cat. no. DP419; Tiangen Biotech, Co., Ltd., Beijing, China). cDNA was synthesized from RNA using a One Step PrimeScript miRNA cDNA Synthesis kit (Takara Bio, Inc., Otsu, Japan). The RT protocol was as follows: $37^{\circ} \mathrm{C}$ for $60 \mathrm{~min}$ and $85^{\circ} \mathrm{C}$ for 5 sec. PCR was subsequently performed using SYBR Premix Ex Taq $^{\mathrm{TM}}$ II (Takara Bio, Inc.) and ABI 7500 Fluorescence Quantitative PCR instrument (Applied Biosystems; Thermo Fisher Scientific, Inc., Waltham, MA, USA) in a $20 \mu \mathrm{l}$ volume. The thermocycling conditions were a follows: $95^{\circ} \mathrm{C}$ for $30 \mathrm{sec}, 40$ cycles of $90^{\circ} \mathrm{C}$ for $5 \mathrm{sec}$ and $65^{\circ} \mathrm{C}$ for $30 \mathrm{sec}$. cDNA (10 $\mu \mathrm{l} /$ lane) was separated in $1 \%$ nucleic acid electrophoresis. $1.2 \%$ agarose (cat. no. A9414; Sigma-Aldrich; Merck KGaA) and $5 \mu \mathrm{l}$ Goodview nucleic acid dye (cat. no. HGV-2; Beijing SBS Genetech Co., Ltd., Beijing, China) were used to separate PCR products, the results of which were observed using a Bio-Rad Gel Imaging Analyzer (Bio-Rad Laboratories, Inc., Hercules, CA, USA). Image J software v1 (National Institutes of Health, Bethesda, MD, USA) was used to analyze gray values. The relative expression of each gene was normalized to $\beta$-actin. Primer sequences were as follows: $\beta$-arrestin 2 forward (F), 5'-ACCCATCACCCTCTGATCCT-3' and reverse (R), 5'-CTGCCCTCTCCTAGTCAGGT-3'; SIRT1 F, 5'-TGC AGGATTTTAGCCCTGGAG-3' and R, 5'-AAAGGATTT TGAGGCAAAAGAAGA-3'; p65 F, 5'-CTGTCCCCAAGC CAGGTAAG and R, 5'-AGAGGTGATTTTTGTTCCCCC A-3'; and $\beta$-actin F, 5'-AAGTACTCCGTGTGGATCGG-3' and R, 5'-TCAAGTTGGGGGACAAAAAG-3'.

Statistical analysis. All data are represented as mean \pm standard deviation. The differences among two groups were analyzed by independent-samples t-tests and multiple groups were compared using one-way analysis of variance followed by Duncan's post-hoc test. Statistical analyses were conducted using SPSS 19.0 (IBM Corp., Armonk, NY, USA). P<0.05 indicated that the difference between groups was statistically significant. 
A
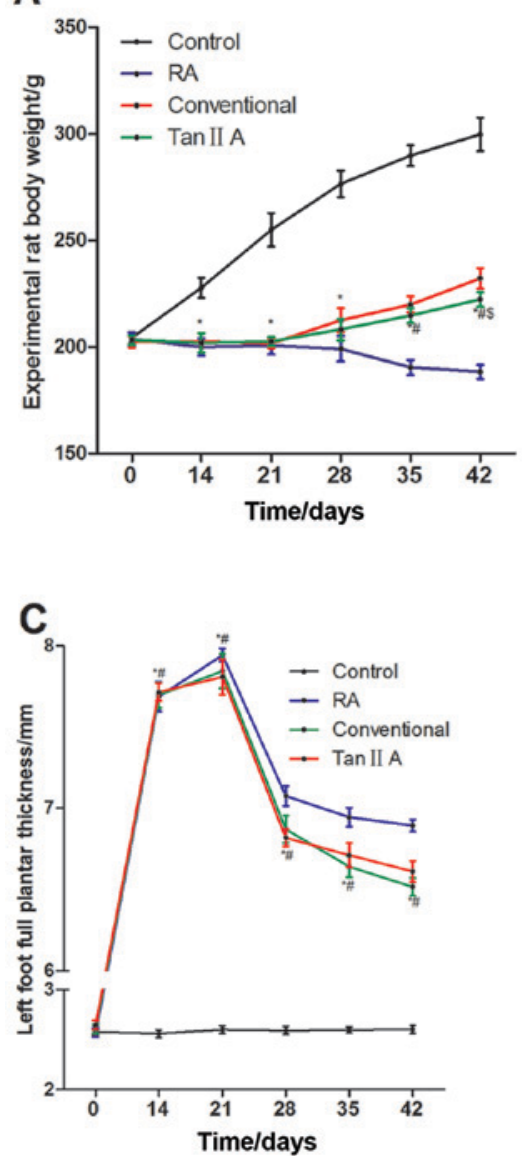

B

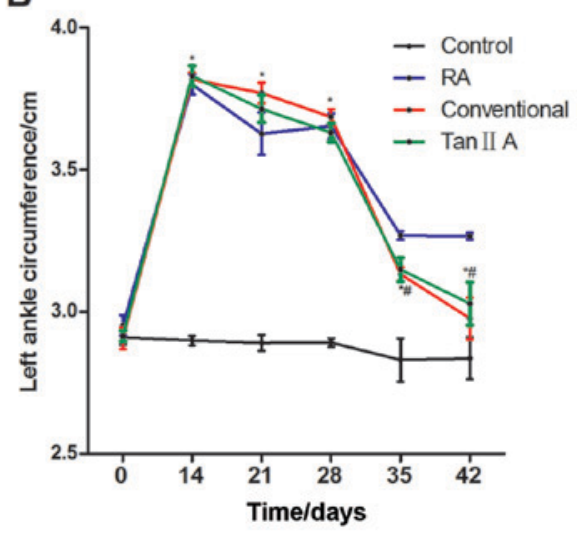

D

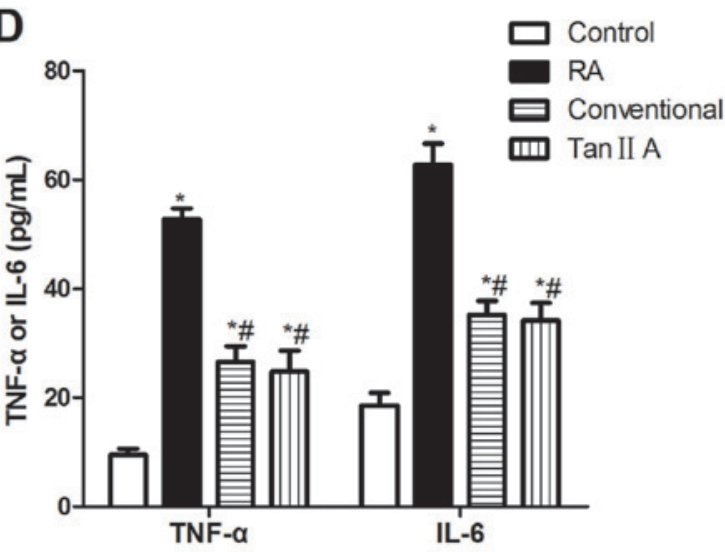

Figure 1. Tan IIA alleviates RA in rats. The (A) body weight, (B) circumference of the left posterior ankle and (C) posterior plantar metatarsal thickness were measured over 42 weeks. RT and Tan IIA treatment were administered 21 days after the establishment of the RA models. (D) TNF- $\alpha$ and IL- 6 protein concentrations after 3 weeks of treatment. ${ }^{*} \mathrm{P}<0.05$ vs. the Control group; ${ }^{\$} \mathrm{P}<0.05$ vs. the RT group. ${ }^{*} \mathrm{P}<0.05$ Tan IIA vs. RA group RA, rheumatoid arthritis; RT, routine treatment (conventional group); Tan IIA, tanshinone II A; TNF, tumor necrosis factor; IL, interleukin.

Table I. TNF- $\alpha$ and IL-6 levels in the serum of patients with rheumatoid arthritis prior to and following treatment.

\begin{tabular}{lcccccc}
\hline & & \multicolumn{2}{c}{ TNF- $\alpha(\mathrm{pg} / \mathrm{ml})$} & & \multicolumn{2}{c}{$\mathrm{IL}-6(\mathrm{pg} / \mathrm{ml})$} \\
\cline { 3 - 4 } Group & Number & Prior to treatment & Following treatment & & Prior to treatment & Following treatment \\
\hline Research & 35 & $45.37 \pm 8.15$ & $38.14 \pm 6.76^{\mathrm{a}, \mathrm{b}}$ & & $8.72 \pm 3.45$ & $4.46 \pm 1.38^{\mathrm{a}, \mathrm{b}}$ \\
Control & 15 & $44.82 \pm 6.97$ & $42.67 \pm 5.42$ & & $8.67 \pm 2.87$ & $8.23 \pm 1.22$ \\
\hline
\end{tabular}

${ }^{\mathrm{a}} \mathrm{P}<0.05$ vs. control group; ${ }^{\mathrm{b}} \mathrm{P}<0.05$ vs. research group prior to treatment. TNF, tumor necrosis factor; IL, interleukin.

\section{Results}

Tan IIA alleviates $R A$ in rats with the condition. No significant difference was identified in the weight of rats in each group prior to the establishment of the RA model (Fig. 1A). On days 14 and 21, rats in the control group were significantly heavier compared with those in the Tan IIA group $(\mathrm{P}<0.05)$. After 1, 2 and 3 weeks of treatment (28, 35 and 42 days after model establishment), the Tan IIA group was significantly lighter compared with the control group $(\mathrm{P}<0.05)$. After 2 and 3 weeks of treatment, the Tan IIA group was significantly lighter compared with the RT group $(\mathrm{P}<0.05)$. After 3 weeks of treatment, the Tan IIA group was significantly heavier compared with the RA group $(\mathrm{P}<0.05)$. The weights of the treated groups were higher compared with those in the RA group.

No significant difference was identified in the circumference of the left posterior ankle of the rats prior to the establishment of the RA model (Fig. 1B). On days 14 and 21 , the circumference in the control group was significantly decreased compared with those in the Tan IIA group $(\mathrm{P}<0.05)$. After 1 week of treatment, the circumference was significantly greater in the Tan IIA group compared with the control group $(\mathrm{P}<0.05)$. After 2 and 3 weeks of treatment, the Tan IIA group was significantly increased and decreased compared with the control and RA groups, respectively $(\mathrm{P}<0.05)$. 

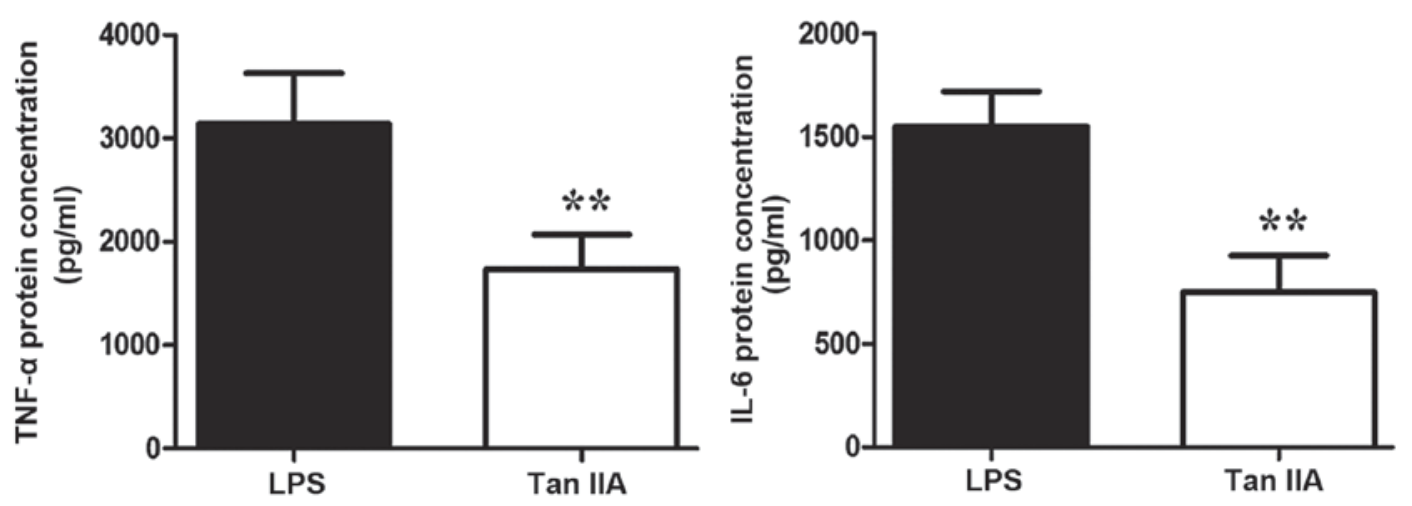

Figure 2. Tan IIA treatment decreases TNF- $\alpha$ and IL-6 levels in peripheral blood mononuclear cells of patients with rheumatoid arthritis. TNF- $\alpha$ and IL-6 protein concentrations after $48 \mathrm{~h}$ of treatment. ${ }^{* *} \mathrm{P}<0.01$ vs. LPS group. Tan IIA, tanshinone II A; TNF, tumor necrosis factor; IL, interleukin; LPS, lipopolysaccharides.
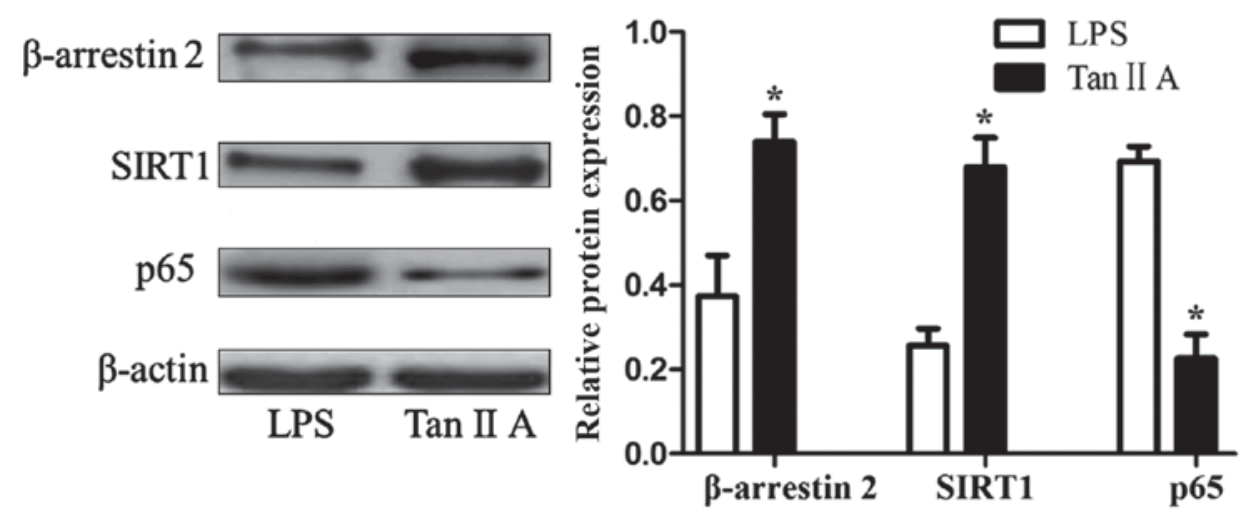

Figure 3. Tan IIA decreases $\beta$-arrestin 2 and SIRT1, and increases p65 proteins in peripheral blood mononuclear cells of patients with rheumatoid arthritis. $\beta$-arrestin 2, SIRT1 and p65 protein expression after $48 \mathrm{~h}$ of treatment. " $\mathrm{P}<0.05$ vs. the LPS group. Tan IIA, tanshinone II A; LPS, lipopolysaccharides; SIRT1, NAD-dependent protein deacetylase sirtuin-1; p65, transcription factor $\mathrm{p} 65$.

No significant difference was identified in the posterior plantar metatarsal thickness of the rats in each group prior to the establishment of the RA model (Fig. 1C). On days 14 and 21, the thickness of the Tan IIA group was significantly greater compared with the control group $(\mathrm{P}<0.05)$. After 1 , 2 and 3 weeks of treatment, the posterior plantar metatarsal thickness in the Tan IIA group was significantly increased and decreased compared with the control and RA groups, respectively $(\mathrm{P}<0.05)$. No significant difference was identified in the posterior plantar metatarsal thickness of the Tan IIA group compared with the RT group.

After 3 weeks of treatment, the concentration of TNF- $\alpha$ and IL-6 in the Tan IIA group was significantly lower compared with those in the RA group $(\mathrm{P}<0.05$; Fig. 1D). No significant difference in protein expression was identified between the Tan IIA and RT groups.

Tan IIA injections decrease TNF- $\alpha$ and IL-6 levels in the serum of patients with $R A$. Prior to treatment, no significant differences in TNF- $\alpha$ and IL-6 levels in the serum of patients with RA were identified between the research and control group (Table I). Following treatment, the TNF- $\alpha$ and IL-6 levels in research group were significantly lower compared with those in the research group prior to treatment and those in the control group following treatment (all $\mathrm{P}<0.05$ ).
Tan IIA treatment decreases TNF- $\alpha$ and IL-6 levels in $P B M C$ s of patients with RA. Tan IIA significantly inhibited the LPS-induced secretion of TNF- $\alpha$ and IL-6 in PBMCs of patients with RA (both $\mathrm{P}<0.01$; Fig. 2).

Tan IIA decreases $\beta$-arrestin 2 and SIRT1, and increases $p 65$ proteins in PBMCs of patients with RA. Tan IIA significantly upregulated the LPS-inhibited expression of $\beta$-arrestin 2 and SIRT1 proteins, and downregulated the LPS-induced expression of the p65 protein in PBMCs of patients with RA (all $\mathrm{P}<0.05$; Fig. 3).

Tan IIA decreases $\beta$-arrestin 2 and SIRT1, and increases $p 65$ $m R N A$ in PBMCs of patients with RA. Tan IIA significantly upregulated the expression of the $\beta$-arrestin 2 and SIRT1 mRNAs in PBMC stimulated by LPS, and inhibited the expression of p65 mRNA compared with cells only stimulated with LPS (all P<0.05; Fig. 4).

$\beta$-arrestin 2 siRNA increases TNF- $\alpha, I L-6$ and $p 65$, and decreases SIRT1 in PBMCs of patients with RA. Compared LPS stimulation for $48 \mathrm{~h}$ alone (A group), TNF- $\alpha$ and IL-6 in PBMCs pretreated with $\beta$-arrestin 2 siRNA for $4 \mathrm{~h}$ (C group) increased significantly. Furthermore, SIRT1 protein expression decreased and p65 protein expression increased (all $\mathrm{P}<0.05$; 

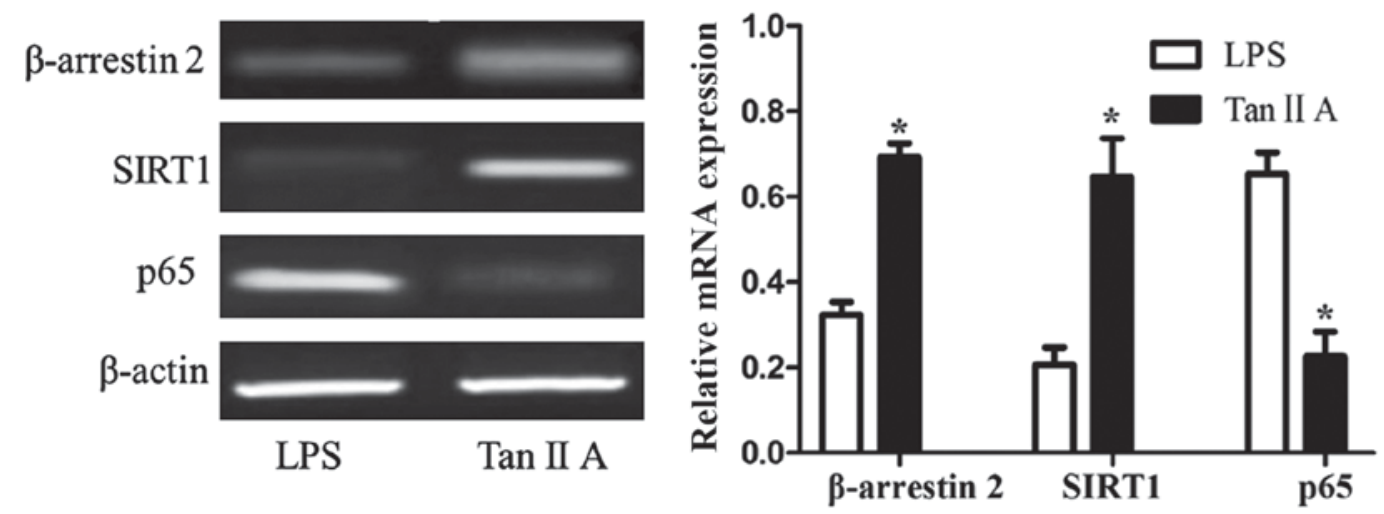

Figure 4. Tan IIA decreases $\beta$-arrestin 2 and SIRT1, and increases p65 mRNA in peripheral blood mononuclear cells of patients with rheumatoid arthritis. $\beta$-arrestin 2, SIRT1 and p65 mRNA expression after $48 \mathrm{~h}$ of treatment. "P<0.05 vs. the LPS group. Tan IIA, tanshinone II A; LPS, lipopolysaccharides; SIRT1, NAD-dependent protein deacetylase sirtuin-1; p65, transcription factor p65.

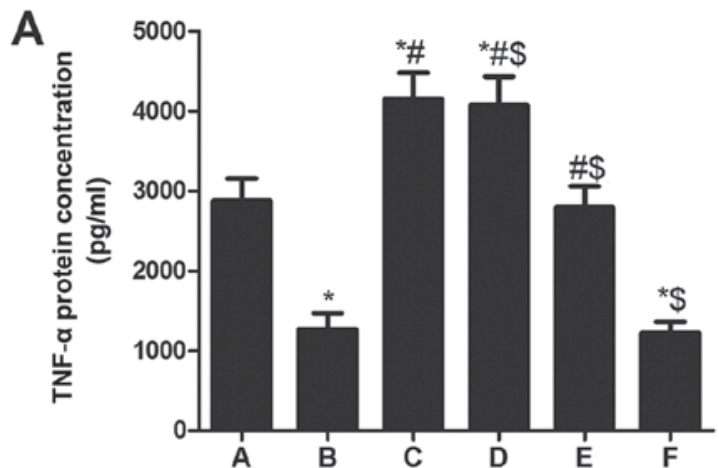

C

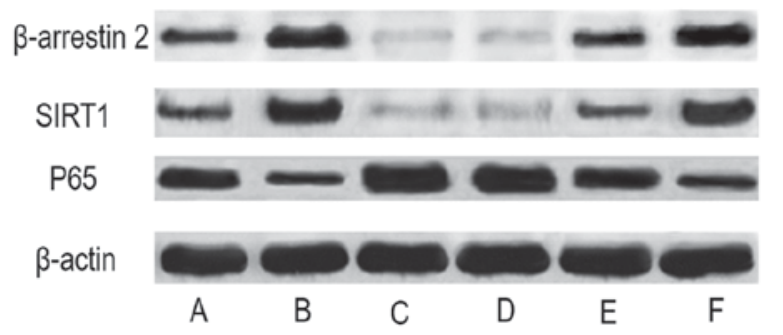

B
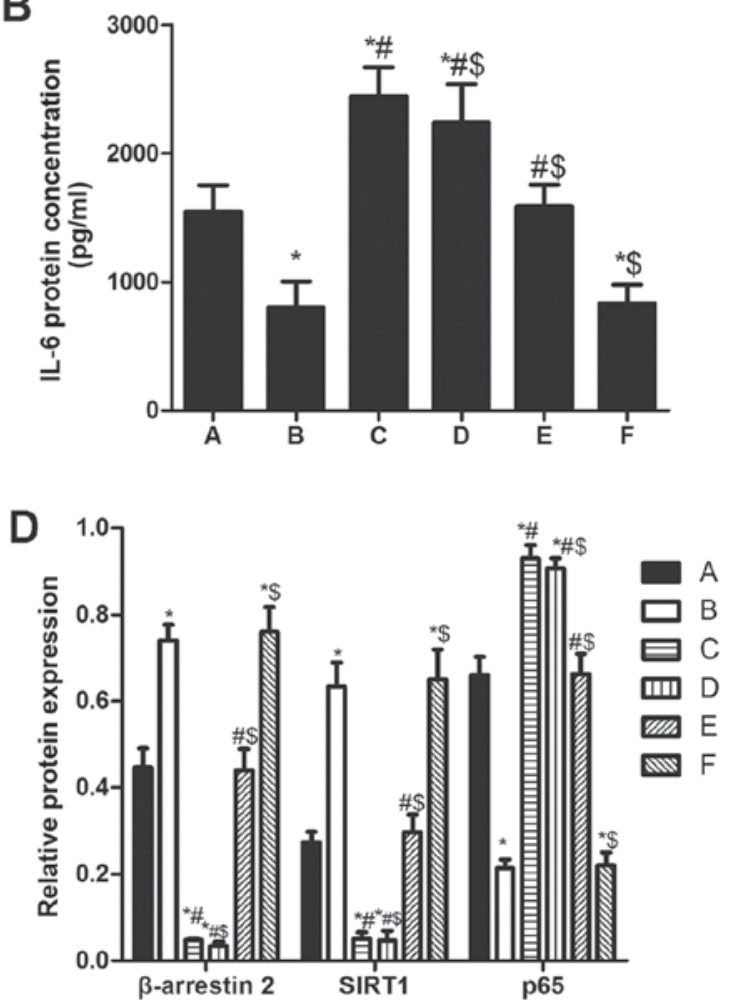

Figure 5. $\beta$-arrestin 2 siRNA increases TNF- $\alpha$, IL-6 and p65, and decreases SIRT1 in peripheral blood mononuclear cells of patients with rheumatoid arthritis. The concentration of (A) TNF- $\alpha$ and (B) IL-6. (C) Qualitative and (D) quantitative analysis of western blotting results for $\beta$-arrestin 2, SIRT1 and P65. The groups were as follows: A, LPS stimulation for $48 \mathrm{~h}$; B, LPS stimulation for $48 \mathrm{~h}$ and Tan IIA treatment for another $24 \mathrm{~h}$; C, $\beta$-arrestin 2 siRNA incubation for $4 \mathrm{~h}$ and LPS stimulation for $48 \mathrm{~h}$; D, $\beta$-arrestin 2 siRNA incubation for $4 \mathrm{~h}$, LPS stimulation for $48 \mathrm{~h}$ and Tan IIA treatment for another $24 \mathrm{~h}$; E, negative control siRNA incubation and LPS stimulation for $48 \mathrm{~h}$; E, negative control siRNA incubation, LPS stimulation for $48 \mathrm{~h}$ and Tan IIA treatment for another $24 \mathrm{~h}$. ${ }^{*} \mathrm{P}<0.05$ vs. A group; ${ }^{*} \mathrm{P}<0.05$ vs. B group; ${ }^{\mathrm{P}} \mathrm{P}<0.05$ vs. C group. si, small interfering; SIRT1, NAD-dependent protein deacetylase sirtuin-1; p65, transcription factor p65; TNF, tumor necrosis factor; IL, interleukin; Tan IIA, tanshinone II A; LPS, lipopolysaccharides.

Fig. 5). Tan IIA did not significantly reverse the $\beta$-arrestin 2 siRNA-induced changes to expression.

\section{Discussion}

TNF- $\alpha$ has been demonstrated to promote the occurrence of inflammation by inducing $\mathrm{T}$ cells to produce a variety of inflammatory factors (1-3). IL-6, a cytokine produced in monocytes, macrophages and lymphocytes, can stimulate and be associated with the immune response in the body by inducing the differentiation of mononuclear cells and B cells, and enhancing the activity of NK cells (1-3). A previous study demonstrated that TNF- $\alpha$ and IL- 6 levels in the serum and synovial fluid of patients with RA were high, indicating that they serve key roles in the pathogenesis of RA by promoting the inflammatory response of patients with RA (2). The current study revealed that TNF- $\alpha$ and IL- 6 levels in the serum of patients with RA in the research group following treatment were significantly 
lower than those in the research group prior to treatment and the control group following treatment, indicating that Tan IIA sulfonate sodium injections could inhibit the inflammatory response in patients with RA. Nizamutdinova et al (13) demonstrated that Tan IIA could inhibit the expression of TNF- $\alpha$ by regulating the phosphatidylinositol 4,5-bisphosphate 3-kinase/RAC- $\alpha$ serine/threonine-protein kinase, protein kinase $\mathrm{C}$ and tyrosine-protein kinase $\mathrm{JAK} /$ signal transducer and activator of transcription (STAT)3 signaling pathways. The aforementioned study also revealed that Tan IIA could inhibit the TNF- $\alpha$-induced ICAM-1 expression. Lin et al (14) revealed that Tan IIA inhibited the in vitro and in vivo growth of breast cancer stem cells by downregulating the IL-6/STAT3/NF- $\kappa$ B signaling pathway, suggesting that Tan IIA regulated the expression of TNF- $\alpha$ and IL- 6 through regulating multiple signaling pathways.

To further reveal the mechanism behind the Tan IIA-induced downregulation of TNF- $\alpha$ and IL- 6 levels, the effect of Tan IIA on the expression of associated proteins (including SIRT1, $\beta$-arrestin 2, TNF- $\alpha$ and IL-6) in LPS-stimulated PBMCs was investigated. The current study demonstrated that Tan IIA inhibited the LPS-induced secretion of TNF- $\alpha$ and IL-6, upregulated the LPS-inhibited expression of $\beta$-arrestin 2 and SIRT1 proteins, and downregulated the LPS-induced expression of p65 protein in PBMCs of patients with RA. However, Tan IIA could not inhibit the $\beta$-arrestin 2 siRNA-induced secretion of TNF- $\alpha$ and IL-6 in PBMCs of patients with RA. These results indicated that Tan IIA inhibited the expression of TNF- $\alpha$ and IL- 6 in patients with RA through upregulating $\beta$-arrestin 2 expression, thus the inflammatory response in patients with RA was inhibited.

$\beta$-arrestin 2 can regulate human immunological functions by inhibiting activation of the $N F-\kappa B$ signaling pathway, and regulating the chemotaxis of immune cells and multiple signaling pathways (1-3). As $\beta$-arrestin 2 serves a key role in regulating human immunological functions, it may be associated with the development and progression of certain autoimmune associated diseases (1-3). Li et al (15) demonstrated that $\beta$-arrestin 2 inhibited RA progression by inhibiting the inflammatory response in RA rats; the authors hypothesized that the inhibitory response may be associated with inhibiting the $\mathrm{NF}-\kappa \mathrm{B}$ signaling pathway. The $\mathrm{NF}-\kappa \mathrm{B}$ signaling pathway is one of the most important signaling pathways in mammalian cells and a node in multiple cell signaling pathways (16). Following its activation, the NF- $\kappa \mathrm{B}$ signaling pathway was revealed to regulate the expression of a variety of downstream inflammatory cytokines, which can regulate the inflammatory response (17).

The current study revealed that $\beta$-arrestin 2 expression in PBMCs of patients with RA was positively associated with SIRT1 expression and was negatively associated with p65. SIRT1 is a histone deacetylase that is widely expressed in human cells (18-20). SIRT1 can deacetylate p53, UCP2, $\mathrm{NF}-\kappa \mathrm{B}$ or other transcription factors to exert biological functions (14-20). p65, a key protein in the NF- $\kappa \mathrm{B}$ signaling pathway, is acetylated to exert its biological functions. SIRT1 can downregulate the acetylation level of the $\mathrm{p} 65$ protein in the inflammatory response, which can inhibit the level of transcription of downstream inflammatory genes, including TNF- $\alpha$ and IL-6 (17). TNF- $\alpha$ and IL-6, as two important inflammatory factors, are not only associated with regulating the body's inflammatory response $(21,22)$, but also serve an important role in the development of rheumatoid diseases $(23,24)$. In summary, the present findings suggested that Tan IIA inhibited NF- $\kappa \mathrm{B}$ activity through upregulating $\beta$-arrestin 2 expression to inhibit the inflammatory response in PBMCs of patients with RA.

\section{Acknowledgements}

Not applicable.

\section{Funding}

No funding was received.

\section{Availability of data and materials}

The datasets used and/or analyzed during the current study are available from the corresponding author on reasonable request.

\section{Authors' contributions}

XW conceived, designed and revised the current study. JT and SZ analyzed the data and wrote the manuscript. FZ analyzed the data. All authors read and approved the final manuscript.

\section{Ethics approval and consent to participate}

The current study was approved by the Ethics Committee of The Third Affiliated Hospital of Zhejiang Chinese Medical University (Hangzhou, China). A written informed consent form was obtained from each patient.

\section{Patient consent for publication}

Not applicable.

\section{Competing interests}

The authors declare that they have no competing interests.

\section{References}

1. Astry B, Harberts E and Moudgil KD: A cytokine-centric view of the pathogenesis and treatment of autoimmune arthritis. J Interferon Cytokine Res Official 31: 927-940, 2011.

2. Mcinnes IB and Schett G: Cytokines in the pathogenesis of rheumatoid arthritis. Nat Rev Immunol 7: 429-442, 2007.

3. Kunz M and Ibrahim SM: Cytokines and cytokine profiles in human autoimmune diseases and animal models of autoimmunity. Mediators Inflamm 2009: 979258, 2009.

4. Simsek I: TNF inhibitors for rheumatoid arthritis-a year in review. Bull NYU Hosp Jt Dis 69: 220-224, 2011.

5. Woodrick RS and Ruderman EM: Interleukin 6 inhibition-RA and beyond. Bull NYU Hosp Jt Dis 69: 225-229, 2011.

6. Kremer JM, Westhovens R, Leon M, Di Giorgio E, Alten R, Steinfeld S, Russell A, Dougados M, Emery P, Nuamah IF, et al: Treatment of rheumatoid arthritis by selective inhibition of T-cell activation with fusion protein CTLA4Ig. N Engl J Med 349: 1907-1915, 2003.

7. Rao DA, Gurish MF, Marshall JL, Slowikowski K, Fonseka CY, Liu Y, Donlin LT, Henderson LA, Wei K, Mizoguchi F, et al: Pathologically expanded peripheral $\mathrm{T}$ helper cell subset drives $\mathrm{B}$ cells in rheumatoid arthritis. Nature 542: 110-114, 2017. 
8. Liu C, Wang D, Lu S, Xu Q, Zhao L, Zhao J, Song Y and Wang H: Increased circulating follicular treg cells are associated with lower levels of autoantibodies in patients with rheumatoid arthritis in stable remission. Arthritis Rheumatol 70: 711-721, 2018.

9. Yu Q, Chen H, Sheng L, Liang Y and Li Q: Sodium tanshinone IIA sulfonate prolongs the survival of skin allografts by inhibiting inflammatory cell infiltration and $\mathrm{T}$ cell proliferation. Int immunopharmacol 22: 277-284, 2014.

10. Shang Q, Wang H, Li S and Xu H: The effect of sodium tanshinone IIA sulfate and simvastatin on elevated serum levels of inflammatory markers in patients with coronary heart disease: A study protocol for a randomized controlled trial. Evid Based Complement Alternat Med 2013: 756519, 2013.

11. Jie L, Du H, Huang Q, Wei S, Huang R and Sun W: Tanshinone IIA induces apoptosis in fibroblast-like synoviocytes in rheumatoid arthritis via blockade of the cell cycle in the G2/M phase and a mitochondrial pathway. Biol Pharm Bull 37: 1366-1372, 2014.

12. Kay J and Upchurch KS: ACR/EULAR 2010 rheumatoid arthritis classification criteria. Rheumatology (Oxford): 51 (Suppl 6): vi5-vi9, 2012.

13. Nizamutdinova IT, Kim YM, Jin H, Son KH, Lee JH, Chang KC and Kim HJ: Tanshinone IIA inhibits TNF- $\alpha$-mediated induction of VCAM-1 but not ICAM-1 through the regulation of GATA-6 and IRF-1. Int Immunopharmacol 14: 650-657, 2012.

14. Lin C, Wang L, Wang H, Yang L, Guo H and Wang X: Tanshinone IIA inhibits breast cancer stem cells growth in vitro and in vivo through attenuation of IL-6/STAT3/NF- $\mathrm{B}$ signaling pathways. J Cell Biochem 114: 2061-2070, 2013.

15. Li P, Cook JA, Gilkeson GS, Luttrell LM, Wang L, Borg KT, Halushka PV and Fan H: Increased expression of beta-arrestin 1 and 2 in murine models of rheumatoid arthritis: Isoform specific regulation of inflammation. Mol Immunol 49: 64-74, 2011.

16. Lan W, Petznick A, Heryati S, Rifada M and Tong L: Nuclear factor- $\kappa \mathrm{B}$ : Central regulator in ocular surface inflammation and diseases. Ocul Surf 10: 137-148, 2012.
17. Zheng $\mathrm{C}$, Yin $\mathrm{Q}$ and $\mathrm{Wu} \mathrm{H}$ : Structural studies of $\mathrm{NF}-\kappa \mathrm{B}$ signaling. Cell Res 21: 183-195, 2011.

18. Thakur BK, Chandra A, Dittrich T, Welte K and Chandra P. Inhibition of SIRT1 by HIV-1 viral protein Tat results in activation of p53 pathway. Biochem Biophys Res Commun 424: 245-250, 2012.

19. Zhao W, Kruse JP, Tang Y, Jung SY, Qin J and Gu W: Negative regulation of the deacetylase SIRT1 by DBC1. Nature 451: 587-590, 2008

20. Kuno A, Hori YS, Hosoda R, Tanno M, Miura T, Shimamoto K and Horio Y: Resveratrol improves cardiomyopathy in dystrophin-deficient mice through SIRT1 protein-mediated modulation of p300 protein. J Biol Chem 288: 5963-5972, 2013.

21. Zelová $\mathrm{H}$ and Hošek $\mathrm{J}$ : TNF- $\alpha$ signalling and inflammation: Interactions between old acquaintances. Inflamm Res 62: 641-651, 2013.

22. Neurath MF and Finotto S: IL-6 signaling in autoimmunity, chronic inflammation and inflammation-associated cancer. Cytokine Growth Factor Rev 22: 83-89, 2011.

23. Seriolo B, Paolino S, Sulli A, Fasciolo D and Cutolo M: Effects of anti-TNF-alpha treatment on lipid profile in patients with active rheumatoid arthritis. Ann NY Acad Sci 1069: 414-419, 2006.

24. Nishimoto N, Hashimoto J, Miyasaka N, Yamamoto K, Kawai S, Takeuchi T, Murata N, van der Heijde D and Kishimoto T: Study of active controlled monotherapy used for rheumatoid arthritis, an IL-6 inhibitor (SAMURAI): Evidence of clinical and radiographic benefit from an $\mathrm{x}$ ray reader-blinded randomised controlled trial of tocilizumab. Ann Rheum Dis 66: 1162-1167, 2007.

This work is licensed under a Creative Commons Attribution-NonCommercial-NoDerivatives 4.0 International (CC BY-NC-ND 4.0) License. 